Post print

\title{
Deliberative academic development: the potential and challenge of agency
}

*Ester Fremstad, University of Oslo, Norway

Andreas Bergh, Örebro University, Sweden

Tone Dyrdal Solbrekke, University of Oslo, Norway

Trine Fossland, University of Troms $\varnothing$-The Arctic university of Norway

To cite this article: Ester Fremstad, Andreas Bergh, Tone Dyrdal Solbrekke \& Trine Fossland (2019): Deliberative academic development: the potential and challenge of agency, International Journal for Academic Development, DOI:

10.1080/1360144X.2019.1631169

*Corresponding author ester.fremstad@iped.uio.no

*ORCID: 0000-0002-9658-8165

This work was supported by the Norwegian Research Council. Project number 246745/H20.

Disclosure statement: There are no conflicts of interest

Data availability statement: Data (audio files and transcripts are available on request)

\section{Acknowledgements}

We are sincerely grateful to the focus group participants for spending the scarce resource that is time to share their ideas and experiences. We also wish to thank the reviewers and the editor for their thoughtful and constructive feedback, as well as colleagues in the Formation project-Molly Supthen in particular-for valuable contributions to earlier versions of this paper.
Abstract
In this paper, we explore possibilities and challenges for deliberative academic development. Deliberative academic development refers to a practice that engages members of the university in dialogue about its purposes, ways of organizing and leading higher education, as well as about teaching and learning. The paper critically analyses data from focus group interviews with academic developers from four universities within two national contexts. Combining sociological conceptualizations of agency and the framework of 'epistemic living spaces,' the paper offers insights into challenges and opportunities for deliberative academic development, as well as a framework for studying agency in other contexts. 
Post print

Keywords: agency, deliberative academic development, epistemic living spaces

\section{Introduction}

It is well documented that the roles and responsibilities of academic developers (ADs) have expanded from a focus on supporting individual teachers to increasingly include organizational development (Schroeder et al., 2011; Stensaker, 2017). The latter includes supporting institutional leaders in adapting institutional practices to political expectations (Debowski, 2014; Gibbs, 2013; Stensaker et al., 2017; Sugrue et al., 2017). This has made the role of academic development more ambiguous in several ways and has brought awareness to the political geography of academic development (Di Napoli, 2014; Holmes \& Manatungha, 2012; Kandlbinder, 2007). In this context, there is a need to widen academic development to include not only enhancing student learning, but engaging with questions about its purposes (Fyffe, 2018; Grant, 2007) and their implications for ways of organizing and leading higher education (Sutherland, 2018).

In the $21^{\text {st }}$ century, universities are expected to serve multiple and sometimes conflicting purposes, some of which contest university traditions. For example, political ambitions to make higher education more entrepreneurial and relevant to the work market, challenge traditional understandings of higher education (Di Napoli, 2014; Karseth \& Solbrekke, 2016; Sutphen, Solbrekke, \& Sugrue, 2018). Positioned in this political space and between university decision makers and faculty, Kandlbinder (2007) argues, ADs must take responsibility for facilitating "active and inclusive debates on student learning... as well as... on the nature if university teaching" (p. 56) with "attention to political events in the world beyond the universities walls" (p. 58). He labels this engaging of and with university members in dialogue about purposes, ways of organizing and leading higher education, and about teaching and learning, 
Post print

'deliberative academic development.' It is this notion of deliberative practice on which we draw in this article.

The present study ${ }^{1}$, in which ADs from four different Scandinavian universities reflect on their roles and responsibilities, gives rise to a similar notion of deliberative practices. Further, as will be demonstrated in the analysis, the ADs explicitly relate this role to current tensions among different educational purposes and approaches, and to ambiguities in a role that is both horizontal and vertical. However, they also report that, in contrast to how they envision their work, deliberative practices play a marginal role in everyday practice. How can this discrepancy between envisioned commitments and current practices be understood? In the following, we explore this as a question of agency, as we aim to understand more about what supports and hinders ADs in making deliberative practices part of their everyday work.

Key underpinning assumptions of the article are that ADs work is influenced considerably by local contexts (Sugrue et al., 2017), and that agency is embedded in specific structural and cultural contexts (Archer, 1996; Emirbayer \& Mische, 1998). Thus, to explore agency, we must capture the relation between ADs local context and their agency. In order to illuminate the possibilities and challenges of deliberative academic development, we therefor ask the following research question:

How is academic developers' agency shaped by and shaping the contexts of academic development?

To explore this question, we combine sociological conceptualizations of agency (Archer, 1996; Emirbayer \& Mische, 1998) and the framework of 'epistemic living spaces' (Felt,

\footnotetext{
${ }^{1}$ The study reported in this paper is part of the international research and competence-building project 'Formation and competence building of University academic developers.' The project includes six institutional partners and is supported by the Norwegian Research Council 2015-2020, project number 246745/H20 (http://www.uv.uio.no/iped/english/research/projects/solbrekke-formation-and-competencebuilding/index.html).
} 
Post print

2009). This combination offers a methodological approach that enables nuanced analyses of the relationship between agency and local context.

\section{Academic development: A demanding position}

Even though local contexts vary, some general demands seem to influence ADs potential agency. Green and Little (2013, p. 535) suggest that ADs' position simultaneously in the margin and between leaders and academics — implies possibilities to act with integrity and substance, to act and not react, to spot contradictions and discrepancies, and to be (more) constructively critical. This position offers opportunities for deliberative academic development. Empirical studies suggest, however, that the position is demanding: It requires expert judgment (Debowski, 2014) that balances meeting expectations to help the institution adapt to political directives, and practices founded on collegiality and traditional academic values (Handal et al., 2014; Roxå \& Mårtensson, 2017). Furthermore, the position requires serving as both horizontal and vertical brokers (Land, 2004; Sugrue et al., 2017) and navigating a complex web of commitments to achieve "legitimate compromises" (e.g. between resistance and policy compliance; Di Napoli, 2014; Handal et al., 2014; Peseta, 2014; Roxå \& Mårtensson, 2017; Saroyan, 2014). This balancing act requires collective approaches among the members of the AD community, both locally and beyond (Solbrekke \& Fremstad, 2018).

The institutions in which ADs work have specific features. Moreover, ADs are positioned in various ways within their organization, and members come from various backgrounds (Sugrue et al., 2017). In the following, we present the analytical lens used to capture how agency is interrelated with specific contextual features. 
Post print

\section{Analytical framework: Agency in epistemic living spaces}

In our analysis, we combine conceptualizations of agency (Archer, 1996; Emirbayer \& Mische, 1998) with the framework of 'epistemic living spaces' (Felt, 2009). While agency is lived out by individuals, it is reliant on collective agency, which is, in turn, embedded in specific structural and cultural contexts (Archer, 1996; Emirbayer \& Mische, 1998). To study agency, we need an approach capable of linking culture and agency while keeping the two analytically distinct to examine their interplay (Archer, 1996). We find that the framework of 'epistemic living spaces', developed by Felt (2009), offers cultural categories appropriate for studying agency (Archer, 1996) within an academic context.

Epistemic living spaces are combinations of actual features of specific contexts; their cultural arrangements; and members' understanding, experience and enactment of them. Thus, members also form these spaces through practices, individual and collective perceptions, and narrative re-construction informed by past and present and oriented toward possible futures (Felt, 2009). Epistemic living spaces can be analyzed according to their temporal, social, epistemic, spatial and symbolic dimensions.

The temporal dimension resonates with Emirbayer and Mische's (1998) emphasis on past, present, and future for understanding agency (i.e. while actors are situated in the present, their practical-evaluative judgments are also related to past experiences and envisioned futures). This dimension helps us understand agency in light of different temporal trajectories of AD units. Agency may be both restricted and enhanced by actors' understandings of future expectations and how these are interpreted in relation to individual and collective pasts. Thus, access to the collective experiences of local and broader communities of ADs is essential (Bergh \& Wahlström, 2018). 
Post print

The interplay between individual and collective experience (e.g. from the broader communities of research and practice in higher education) are central to understand challenges and possibilities for agency. The social dimension captures forms of togetherness and belonging to these communities, which imply access to different sources of knowledge and experiences and is, thus, central to the epistemic dimension. This dimension also includes the fact that universities are constituted by various ‘epistemic cultures' (Knorr Cetina, 1999), 'tribes and territories' (Becher \& Trowler, 2001), and professional groups (i.e. faculty, administration, management, and leadership). Various forms of togetherness (or not) with these groups imply different epistemic and symbolic grounds for agency.

The spatial dimension is useful to identify how AD units are positioned within organizations as well as other spatial features relevant to their agency. Central to ADs' epistemic living space is that they also encompass their institutional roles, selfperceptions, and how they are, and experience being perceived by other university actors. This is captured in the symbolic dimension.

The dimensions of epistemic living spaces are inextricably intertwined, but distinguished for analytical purposes. It is insights into how features of the different dimensions are interrelated that provide understanding of, in this case, academic developers' agency and how it is shaped by, but also (capable of) shaping specific conditions within the given contexts.

\section{Method}

In this study, we draw on focus group interviews with ADs from the academic development units of two Norwegian and two Swedish public universities. To analyze the interviews in relation to the ADs' contexts, we draw also on authors' insights into these contexts due to appointments within these four universities, as well as findings 
Post print

from other parts of the research project (Sugrue et al., 2017; Sugrue et al., forthcomming; Sutphen, Solbrekke, \& Sugrue, 2018).

During fall 2016, we conducted semi-structured focus group interviews with three to six ADs from each of the four units. In total, 17 ADs participated. The study is approved by the Norwegian Centre for Research Data ${ }^{2}$. Ethical considerations were important throughout the study in terms of information to participants, recruitment, privacy, and presentation of participants and units. All ADs within the four institutions were informed about the project and invited to participate. Participation was voluntary and depended on their ability to attend at scheduled times. The participants were all active members of their units and included both newcomers and experienced members. The interviews lasted approximately 90 minutes and were performed in the ADs' native languages by two of the authors. All participants approved that the interviews were recorded and transcribed verbatim.

Focus group interviews encourage negotiation of meaning, but also invite different views (Morgan, 1996) and experiences (Chiongel et al., 2003). Participants were encouraged to talk about their practices, work conditions, expectations from teachers and leaders, their responses to these, and relations to and conceptions of their universities' strategic documents. This yielded information about their current work, how they experience their work contexts, past experiences, and aspirations for the future.

Two of the authors hold positions as ADs within the studied units and took part as informants in the interviews at their local units. To handle inevitable bias, authors external to these units took responsibility for analyzing the data from these contexts. Our insider-outsider approach ensured important contextual insight, yet also the necessary critical distance when preparing, conducting, and analyzing the interviews

\footnotetext{
${ }^{2}$ Project reference 45852 .
} 
Post print

(Dwyer \& Buckle, 2009; Sutphen, Sugrue, \& Solbrekke, 2018). At one stage, the participants reviewed the analysis and quotations and offered clarifications. The analysis and translation of quotes is, however, the responsibility of the authors.

The analysis developed through an iterative process of interpretation and reinterpretation of transcripts and theory, with one lending meaning to the other (Alvesson \& Sköldberg, 2009). Through the process of interpretation, where we explored data and theory simultaneously, specific features of the different contexts emerged as important for agency. By identifying how temporal, social, epistemic, spatial and symbolic features influence agency in interrelated ways, we portray how different epistemic living spaces present different opportunities and challenges for practicing deliberative academic development.

\section{Contextualizing the study}

The four public universities in which the AD units are situated - University of Oslo (UiO), University of Troms $\varnothing-$ the Arctic University of Norway (UiT), Uppsala University (UU), and Örebro University (ÖU) — have similarities but also differences. UiO and UU are broad, research-intensive universities with long traditions. They were the first universities in their national contexts, established in 1477 and 1811, respectively. UiT and ÖU, both founded in the 1960s, have considerably shorter histories.

All four AD units serve faculties across their universities. They provide university pedagogy courses obligatory for newly appointed faculty. Additionally, all units offer consultation for faculties and educational leaders across their universities. However, the AD units' organizational location, histories, and recruitment policies differ significantly. 
Post print

The $\mathrm{AD}$ unit at $\mathrm{UiO}$ is placed within the academic line and organizationally, as well as physically located at the Department of Education, Faculty of Educational Sciences. The unit was formed in the 1960s in response to numerous faculty contacting the Department of Education for help with their teaching. The unit consists of seven ADs in academic positions (50\% research and 50\% teaching) recruited from educational science, IT science (one), and political science (one). Additionally, the unit has a fulltime position for administrative support. Most of the ADs have several years of experience within the unit.

At UiT, the unit where ADs have their appointments is part of the university library, which is in the administrative line. The unit consists of ten people working with digital technology, five ADs with academic positions (50\% research and 50\% teaching), two $\mathrm{Ph} . \mathrm{D}$. students, and a $40 \%$ position for administrative support. The ADs are recruited from educational sciences, psychology, and sociology. The AD unit is fairly young and has been gradually built since 2006 .

In contrast to the Norwegian cases, in the Swedish cases, all ADs hold administrative positions and are organizationally placed in the administrative line. Thus, while they have academic backgrounds, they are not allocated time for research. At UU, the AD unit was formed in 1990 and has been organizationally located within the Division for Quality Enhancement since 2011/2012. The unit consists of 27 people, of whom 17 work as ADs. ADs are recruited from various disciplines, and while nine work as ADs full-time, the rest divide their time between the unit and their "home" departments.

The AD unit at ÖU is organizationally located in the Office for Academic Policy, a central administrative unit reporting to the university director. The unit was established by the University Board in 2013. It consists of seven people: one ICT 
Post print

pedagogue; two administrators who provide support for IT systems; one administrative coordinator; and three ADs recruited from various disciplines.

It is clear that the spatial, temporal, and epistemic dimensions of the four units differ. Below, we analyze and critically discuss how agency to enact deliberative academic development is interrelated with context-specific features.

\section{Agency within different epistemic living spaces}

With a particular focus on the agency important for enacting deliberative practices, we have analyzed the reflections on academic development work articulated in the interviews in light of the temporal, social, epistemic, spatial and symbolic dimensions of epistemic living spaces. In the following we portray four epistemic living spaces which foster and hinder this agency in specific ways.

\section{Oslo: Maintaining established collegiality and credibility}

The extensive tradition of the AD unit at the University of Oslo (UiO) is central to its epistemic living space. The unit is both organizationally and physically located at the Department of Education, where ADs hold academic positions. In the interviews, these temporal and spatial features emerge as crucial for ADs' acknowledgement among and collegial relationship with faculty, fostering their agency to engage in deliberative dialogues with them. The symbolic importance of the location was emphasized through contrasts with an administrative position: "I believe that if we were perceived as an administrative unit, we would probably be met differently... we would risk a change in our legitimacy" (UIO-1). Furthermore, "we do not want to be perceived as an instrument for implementing leaderships' decisions" (UiO-2). The ADs' current position was described as essential for how ADs are perceived among leaders and faculty and for their belonging to the academic community. This feature of the social 
Post print

dimension, positioning ADs as insiders, seems to support their agency to initiate deliberative discussions.

UiO's AD unit has a long history of supporting teachers through courses and consultations and of holding a seat in the university's strategic educational committee. The unit's extensive past is important for its temporal trajectory: It implies access to extensive collective experiences and has laid long foundations of credibility among teachers and leaders. The unit has also engaged in the initiation and development of academic development as a field of international practice and research, yielding access to networks such as the International Consortium for Educational Development. Also, as researchers within higher education, the ADs draw on an extensive tradition of research-based knowledge. Thus, this temporal trajectory implies a broad repertoire of knowledge and experiences crucial for the epistemic basis of their work, which has, in turn, symbolic importance. As found in interviews with institutional leaders (Sugrue et al., forthcoming), these leaders conceive of ADs as credible and important institutional actors. This temporal trajectory, therefore, supports agency through an extensive collective epistemic foundation and recognition within the institution.

This epistemic living space seems like a solid ground for agency, and the ADs' descriptions of how they work, in many ways, suggest significant agency. However, practices associated with deliberative academic development, e.g. "discussing with the course participants "what kind of candidates do we educate?" [UiO-1] and "what they see as the purpose of their educational practice" [UiO-4]) are "not part of everyday practice (...) these are not topics that we touch upon in our courses" (UiO-4).

With a solid temporal trajectory, epistemic basis, and reputation within the institution, what prevents this unit from pursuing envisioned deliberative academic development? We suggest that the contextual features that strengthen ADs' position 
Post print

may also hinder their agency to take on deliberative practices. Despite the ADs' acknowledged and collegial position within the institution, this position requires constant maintenance. This may prevent their pursuit of deliberative practices: Upholding practices already acknowledged by teachers and leaders may be (seen as) crucial for maintaining their position. It may also be challenging to combine a "wellnurtured" collective memory of responding to teachers' needs with the agentic, proactive, and critical elements of deliberative practices.

\section{Tromsø: Striving to be perceived as researchers while serving a distributed university}

The University of Troms $\emptyset-$ The Artic University of Norway (UiT) is distributed across ten campuses spread across a vast geographical area, and ADs travel to the various campuses to perform their work. This result in their resources being spread thin and thus lack of time to discuss and pursue envisioned deliberative practices. In this way, the distributed landscape hinders ADs' agency.

The AD unit is organizationally located within the university library, a community with which they "are not really related" (UiT-3). Furthermore, the ADs describe this location as challenging their position within the university because they depend on "... understanding research and basing our practice on research — otherwise, our work will not be taken seriously" (UiT-3). The current location was seen to hinder other institutional actors' perceptions of the ADs as researchers: "I don't think we are perceived as a place where research takes place" (UiT-2). These quotes underscore the relation between the spatial and symbolic dimensions and illustrate how location can undermine important symbolic aspects of agency.

Located in the far north, the unit is separated from other ADs by geographical distance. However, rather than isolating themselves "up here in the far north" (UiT-1), they "have engaged in the International Society for the Scholarship of Teaching and 
Post print

Learning (ISSOTL)" (UiT-1) as well as "travelled for inspiration... and to network" (UiT-1). They have partaken in both national and international communities, gaining access to a broader repertoire of experiences. This is important for the epistemic, social, and symbolic dimensions of their epistemic living space: In addition to providing access to an important epistemic knowledge base, it implies contributing to this knowledge base and belonging to a broader community of practice and research, which serves symbolic purposes of recognition among faculty and leaders within the institution and beyond. This interplay among epistemic, social, and symbolic dimensions seems to support the ADs' agency and illustrates how enacting agency to engage in communities beyond the institution has influenced their epistemic living space.

The ADs at UiT described including deliberative reflections on student formation in their courses, discussing, for example "how the use of technology contributes to students' learning and formation", and in terms of students "not merely obtaining practical skills," but also acquiring "a broad perspective and becoming a world citizen" (UiT-3). The ADs discussed this in terms of generic skills and the question of how to create study programs that foster such skills in a context where modularization of educational programs threatens the focus on student formation. However, apart from having recently "included this issue in a project on educational leadership" (UiT-3), this is described as having been largely unattended: "we have not come very far (...) we never get around to discussing it" (UiT-3).

Given the unit's brief history, it has had little time to establish recognition, credibility, and a collective repertoire of experiences necessary for agency. For example, unit has been unable to protect time for research, and for critically discussing how to balance the present focus on answering external demands to "effectively deliver courses" (UiT-2) with critical and deliberative practices. 
Post print

\section{Uppsala: Defending academic values from an administrative position}

ADs at Uppsala University (UU) express strong affiliation with UU's long-established academic tradition. Deliberative practices, such as critically discussing "the view of the academy: What are its purposes? What kind of citizens do we want to foster in the university?" (UU-2) with faculty, emerged as a core commitment. Additionally, the ADs noted a responsibility to critically address "what kind of structure and organization can enable [these purposes]" (UU-2) and, thus, for vertical brokering in a role as codefenders of traditional academic values, such as "academic freedom, critical and independent thinking" (UU-2) and "Bildung" (UU-4) in a time emphasizing “employability" (UU-2) and "predefined learning outcomes" (UU-4). However, given their current organizational location within the Department for Quality Enhancement, they feared being associated with management, which "challenges collegiality" (UU-4) with faculty, which they considered an important "foundation" (UU-4) for nurturing deliberative discussions:

...organizationally, this meant a step further into management... and that may be problematic, related to those we meet in courses and consultations, because we want to nurture independent and unconditional discussions, and not give the impression that we are part of an administrative and hierarchical organization (UU-4).

The quotes demonstrate how the spatial feature of organizational location necessitates the question about "our role in the organization" (UU-2). This poses challenges for agency along the social and symbolic dimensions because it threatens faculty's perceptions of ADs as colleagues. However, being closer to institutional management and "mov[ing] between management and core [academic] activities" is seen as "both a challenge and an asset" (UU-4): While it may challenge the collegiality with faculty crucial for horizontal brokering, being close to leadership may strengthen agency for vertical brokering. 
Post print

In addition to an ambiguous organizational location posing possibilities but also challenges to agency, ADs at UU is a diverse group, recruited from a variety of disciplines, and many share their time between their home discipline and academic development, thus perform research not as ADs but within their respective disciplines. This implies that their epistemic togetherness is restricted. Though the AD unit has 25 years of experience on which to base a shared set of commitments, in the interviews, the ADs often referred to the general academic values and traditions of UU, expressing a strong commitment to these, while rarely articulating more specific commitments as ADs. While these values and commitments may very well cohere, we interpret this as a relatively weak sense of togetherness among the ADs as a group. This may hinder collective agency based on research and a collective past as ADs'. For example, this may explain why they have not yet managed to be "more in the forefront, as someone who leads development based on the newest developments within the field of education, [addressing] what knowledge and learning means in complex times" (UU-5). On the other hand, epistemic heterogeneity supports an awareness "of the different values and traditions in different parts of the university" (UU-1), offering both symbolic and epistemic assets for agency to engage in deliberative practices.

\section{Örebro: Enacting a role clearly defined by institutional leadership}

The ADs at Örebro University (ÖU) inhabit an epistemic living space characterized by a clearly defined role assigned by institutional leadership, a brief history, and an organizational position within the central administrative unit reporting to the university director.

The unit "was very clearly defined when it was established... and it has been a priority for institutional leadership" (ÖU-1). The ADs' role is to "ensure that existing educational philosophy and strategies are conveyed outwards... visions, objectives and 
Post print

strategies should work its way down the line and we're part of that line" (ÖU-4). ADs at ÖU seemed to locate their legitimacy largely in their clear mandate to "provide competence building for the teachers" (ÖU-3). Furthermore, they experienced that they "need [perspectives] to come from above, that they [leadership] say it is important, because otherwise, we can stand there and talk about these concepts and try to get them across, and they [teachers] just wave them off' (ÖU-4). These quotes suggest little sense of agency, due partially to the unit's symbolic positioning in a hierarchical line with a focus on vertical brokering and partially to a lack of recognition among faculty.

However, ADs identified tensions in their role, voicing a more critical and deliberative approach to academic development:

The focus is becoming very oriented toward educating skilled competence... the new public management idea and Bologna... What is it that we lose in this approach to higher education? In courses, we talk about this educational ideal. What do we include? What don't we include? I believe there is a need to reflect on how to educate citizens who can be critical and defend democracy and human rights... this may sound pretentious, but hopefully we educate students who will contribute to societal development with a critical approach and capacities to handle insecurity (ÖU-3).

The quote presents a critical perspective on current trends in higher education. Further, ADs stress a deliberative practice in relation to the question about purposes; "it is important with regard to our role, to challenge teachers: 'what does this look like in educational practice?"'(ÖU-1). Current policies and priorities are acknowledged as something "we have to relate to, but... we still have this room to maneuver" (ÖU-3). The following quote emphasizes the critical and epistemic foundation of deliberative practice.

"if I can be very visionary, then I think that we really have an opportunity to raise these discussions in the courses, to create an open environment; 'it's okay to be critical here but, but be so on an informed basis' (...) The field of teaching and learning in higher education can contribute a scientific approach, so that we have research and science as a base for what we do" (ÖU-3). 
Post print

While the ADs described deliberative practices as important, as in the other cases, it was expressed more as a vision than as current and shared practice. The lack of a collective epistemic foundation emerged as central to understanding what hinders agency for ADs at ÖU. These ADs are not researchers within higher education; thus, they lack a shared epistemic basis, which the above quote and analyses of other contexts demonstrate to be a crucial part of deliberative discussions.

Further, our analysis suggests that, within ÖU's young AD unit, ADs do not (yet) have a collective repertoire of experiences on which to draw in defining their role and commitments. Having had little time to establish distance from leadership and hierarchical structures and develop collegial relationships with faculty, the ADs' present orientation focuses largely on settling into a new unit by fulfilling vertical expectations and institutionally defined mandates.

\section{Discussion}

Approaching the participants' descriptions through a combination of sociological conceptualizations of agency and the framework of epistemic living spaces, we have systematically addressed the relation between context and ADs' agency within four different universities, including both old and young institutions within two national contexts. The study contributes nuances to our understanding of how multiple features of ADs' contexts may facilitate or hinder the agency to practice deliberative academic development.

In particular, we have demonstrated how temporal trajectories and organizational location support and restrain agency through the way that these features are interrelated with epistemic, social, and symbolic dimensions. Further, the findings illustrate the importance of a shared epistemic living space for possible collective agency. As Bergh and Wahlström (2018) have shown, “an individual teacher's repertoire of agency is not 
Post print

just a question of individual experiences, but is also a question of individual teachers' access to the profession's collective experience base" (p. 14).

The interviewed ADs reported building and maintaining collaborative and collegial relationships with faculty as crucial for their work. They further noted that this requires appropriate distance from hierarchical structures to be perceived as acting with professional integrity and not as representatives of senior leaders. The different epistemic living spaces of the units partaking in this study provide examples of different opportunities for ADs to balance vertical and horizontal roles and, thus, for inhabiting and enacting this 'zone of proximal distance' from leadership and managerial structures (Sugrue, 2015) to develop and enact agency. At the same time, deliberative academic development includes bridging between leaders, management and academic staff in order to facilitate conversations about the purposes of higher education and what these purposes imply for teaching as well as for ways of organizing and leading higher education. The present paper, as well as previous studies (e.g. Karseth \& Solbrekke 2016), suggest that such discussions and thus important educational purposes are currently marginalized.

Some features of ADs' epistemic living spaces, such as temporal trajectory, recruitment, and organizational location may be difficult to influence in the short term. However, the analysis draws attention to features that ADs (may) proactively develop in order to collectively define and enact deliberative academic development. While ADs' work must reflect the needs and aspirations of teachers and leaders, the reported sense of membership within the university suggests that ADs could and should play a more proactive role in defining these needs and aspirations. As Fyffe (2018) argues, enhancing the role of academic development in this sense requires that ADs collectively and individually attend to the 'idea of the university.' Agency should be solidly based 
Post print

within a trajectory of higher education that includes profound insights into both past and present, and engages in research-based perspectives on the (future) purposes of higher education and its contributions to society. This has implications for development along the epistemic dimension: As Sutherland (2018) proposes, the current landscape requires more holistic epistemic approaches. Our findings suggest a need to broaden the scope of ADs' research base to include not only teaching and learning, but also the political and institutional level of higher education and the values and purposes of past, current, and future higher education. A lack of shared reflections on these issues and their implications for academic development may be part of the reason deliberative academic development are discussed in very general and visionary terms and included only to a limited degree in ADs' concrete everyday practices. Shared repertoires of knowledge and approaches are important for both the unit's collective agency and the individual AD.

While the past influences agency in important ways, ADs have also the opportunity to engage in "narrative reconstructions of their epistemic living spaces" (Felt, 2009), including formulations of envisioned futures. Such reconstructions may strengthen their agency to develop capacities "to make practical and normative judgments among alternative possible trajectories of actions, in response to emerging demands, dilemmas, and ambiguities" and the "imaginative generation of possible future trajectories" (Emirbayer \& Mische, 1998, p. 71). The ADs participating in the present study engaged in such narrative reconstruction in the interviews, but reported rarely discussing it within their units. Paradoxically, ADs envision challenging and supporting teachers to pursue important educational purposes, while reporting lack of time and external expectations hindering them in pursuing their own envisioned commitments. Knowledge about cultural features that enable and hinder agency 
Post print

developed through this study may help ADs collaboratively define and perform the roles and responsibilities they find important. Future studies of ADs' practices, particularly among ADs aiming to enact deliberative academic development, can provide important knowledge supporting this aim.

\section{Biographies}

Ester Fremstad is senior lecturer, academic developer, and educational researcher at the University of Oslo, Department of Education. Her research interests include the societal role and responsibility of higher education institutions, teaching for social responsibility, as well as the professional responsibility of academic developers.

Andreas Bergh is Associate professor and Senior Lecturer of Education at Örebro University. His research interest is within the field of curriculum theory with a specific interest in education policy, issues of quality and the professional roles of teachers and school leaders. He is currently engaged in studies of higher education, segregation and of teachers' anti-racist actions.

Tone Dyrdal Solbrekke is professor at University of Oslo, Department of Education. Her research interest spans wide and comprises several qualitative studies on professional responsibility, leadership in higher education and the formative aspect of institutional leading and teaching practices. She is the project leader of the Formation project referred to in this article.

Associate professor Trine Fossland's area of research is within the field of higher education, with a specific interest in digital technology, academic development and educational leadership. She is currently deputy chair of the Norwegian Network for Academic Developers. 
Post print

\section{References}

Alvesson, M., \& Sköldberg, K. (2009). Reflexive methodology: New vistas for qualitative research. London: Sage Publications.

Becher, T., \& Trowler, P. R. (2001). Academic tribes and territories: Intellectual enquiry and the cultures of discipline. Buckingham \& Philadelphia: The Society for Research into Higher Education and Open University Press.

Bergh, A. \& Wahlström, N. (2018). Conflicting goals of educational action - a study of teacher agency from a perspective of transactional realism.. Curriculum Journal 29(1), 134-149.

Chiongel, N. E., Van Der Veen, R. G. W., Wildemeersch, D., \& Jarvis, P. (2003). The validity and reliability of focus groups as a research method in adult education. International Journal of Lifelong Education, 22(5), 495-517. doi:10.1080/0260137032000102850

Debowski, S. (2014). From agents of change to partners in arms: The emerging academic developer role. International Journal for Academic Development, 19(1), 50-56.

Di Napoli, R. (2014) Value gaming and political ontology: Between resistance and compliance in academic development. International Journal for Academic Development, 19(1), 4-11. doi:10.1080/1360144X.2013.848358

Dwyer, S. C., \& Buckle, J. L. (2009). The space between: On being an insider-outsider in qualitative research. International Journal of Qualitative Methods, 8(1), 5463.

Emirbayer, E., \& Mische, A. (1998). What is agency? The American Journal of Sociology, 103(4), 962-1023. 
Post print

Felt, U. (Ed.). (2009). Knowing and living in academic research: Convergence and heterogeneity in research cultures in the European context. Prague: Academy of Sciences of the Czech Republic.

Fyffe, J. M. (2018). Getting comfortable with being uncomfortable: A narrative account of becoming an academic developer. International Journal for Academic Development, 23(4), 355-366. doi:10.1080/1360144X.2018.1496439

Gibbs, G. (2013). Reflections on the changing nature of educational development. International Journal for Academic Development, 18(1), 4-14. doi:10.1080/1360144X.2013.751691

Grant, B. (2007). The mourning after: Academic development in a time of doubt. International Journal for Academic Development, 12(1), 35-43.

Green, D. A., \& Little, D. (2013) Academic development on the margins. Studies in Higher Education, 38(4), 523-537. doi:10.1080/03075079.2011.583640

Handal, G., Lycke, K. H., Mårtensson, K., Roxå, T., Skodvin, A., \& Solbrekke, T. D. (2014). The role of academic developers in transforming Bologna regulations to a national and institutional context. International Journal for Academic Development, 19(1), 12-25. doi:10.1080/1360144X.2013.849254

Holmes, T., \& Manathunga, C. (2012). Of passports, maps, and suitcases: Geopolitical metaphors in academic development. International Journal for Academic Development, 17(3), 193-195. doi:10.1080/1360144X.2012.701086

Kandlbinder, P. (2007). The challenge of deliberation for academic development. International Journal for Academic Development, 12(1), 55-59. doi:10.1080/13601440701217345

Karseth, B., \& Solbrekke, T. D. (2016). Curriculum trends in European higher education: The pursuit of the Humboldtian University Ideas. In S. Slaughter \& J. 
Post print

T. Barrett (Eds.), Higher education, stratification, and workforce development: Competitive advantage in Europe, the US, and Canada (pp. 215-233).

Cham: Springer.

Knorr Cetina, K. (1999). Epistemic cultures: How the sciences make knowledge. Cambridge, MA: Harvard University Press.

Land, R. (2004). Educational development: Discourse, identity and practice.

Maidenhead: Open University Press/Society for Research into Higher Education

Little, D., \& Green, D. A. (2012). Betwixt and between: Academic developers in the margins. International Journal for Academic Development, 17(3), 203-215. doi:10.1080/1360144X.2012.700895

Morgan, D. (1996). Focus groups. Annual Review of Sociology, 22, 129-152.

Peseta, T. L. (2014). Agency and stewardship in academic development: The problem of speaking truth to power. International Journal for Academic Development, 19(1), 65-69. doi:10.1080/1360144X.2013.868809

Roxå, T., \& Mårtensson, K. (2017). Agency and structure in academic development practices: Are we liberating academic teachers or are we part of a machinery suppressing them? International Journal for Academic Development, 22(2), 95105. doi:10.1080/1360144X.2016.1218883

Saroyan, A. (2014). Agency matters: Academic developers' quests and achievements. International Journal for Academic Development, 19(1), 57-64. doi:10.1080/1360144X.2013.862622

Schroeder, C. M., Blumberg P., Van Note Chism, N., Frerichs, C.E., Gano-Phillips, S. \& Rosier, T. (2011). Coming in from the margins: Faculty development's 
Post print

emerging organizational development role in institutional change. Sterling: Stylus Publishing.

Stensaker, B., Van der Vaart, R., Solbrekke, T. D., \& Wittek, A. L. (2017). The expansion of academic development: The challenges of organizational coordination and collaboration. In B. Stensaker, G. Bilbow, L. Breslow, \& R. Van der Vaart (Eds.), Strengthening teaching and learning in research universities: Strategies and initiatives for institutional change (pp. 19-42). New York: Palgrave Macmillan.

Sugrue, C. (2015). Unmasking school leadership a longitudinal life history of school leaders. Dordrecht: Springer.

Sugrue, C., Englund, T., Solbrekke, T. D., \& Fossland, T. (2017). Trends in the practices of academic developers: Trajectories of higher education? Studies in Higher Education. 43(12), 2336-2353. doi:10.1080/03075079.2017.1326026

Sugrue, C., Solbrekke, T. D., Bergh, A., \& Fossland, T. (forthcoming). University Leaders' Talk about Institutional Missions and Academic Developers' Contributions. European Educational Research Journal.

Sutherland, K. A. (2018). Holistic academic development: Is it time to think more broadly about the academic development project? International Journal for Academic Development, 23(4), 261-273. doi:10.1080/1360144X.2018.1524571

Sutphen, M. P., Solbrekke, T. D., \& Sugrue, C. (2018). Toward articulating an academic praxis by interrogating university strategic plans. Studies in Higher Education, 43(1), 1-13 doi:10.1080/03075079.2018.1440384 